University of New Hampshire

University of New Hampshire Scholars' Repository

Space Science Center

Institute for the Study of Earth, Oceans, and

Space (EOS)

2000

\title{
The development of a position-sensitive CZT detector with orthogonal co-planar anode strips
}

\author{
K Larson \\ University of New Hampshire - Main Campus \\ L A. Hamel \\ University of Montreal \\ V T. Jordanov \\ Digirad \\ John R. Macri \\ University of New Hampshire - Main Campus, John.Macri@unh.edu \\ Mark L. McConnell \\ University of New Hampshire - Main Campus, mark.mcconnell@unh.edu
}

See next page for additional authors

Follow this and additional works at: https://scholars.unh.edu/ssc

Part of the Astrophysics and Astronomy Commons

\section{Recommended Citation}

The development of a position-sensitive CZT detector with orthogonal co-planar anode strips Larson, $\mathrm{K}$. A. and Hamel, L. and Jordanov, V. and Macri, J. R. and McConnell, M. L. and Ryan, J. M. and Tousignant, 0. and Vincent, A., AIP Conference Proceedings, 510, 774-778 (2000), DOl:http://dx.doi.org/10.1063/ 1.1307035

This Conference Proceeding is brought to you for free and open access by the Institute for the Study of Earth, Oceans, and Space (EOS) at University of New Hampshire Scholars' Repository. It has been accepted for inclusion in Space Science Center by an authorized administrator of University of New Hampshire Scholars' Repository. For more information, please contact Scholarly.Communication@unh.edu. 


\section{Authors}

K Larson, L A. Hamel, V T. Jordanov, John R. Macri, Mark L. McConnell, James M. Ryan, O Tousignant, and A Vincent 


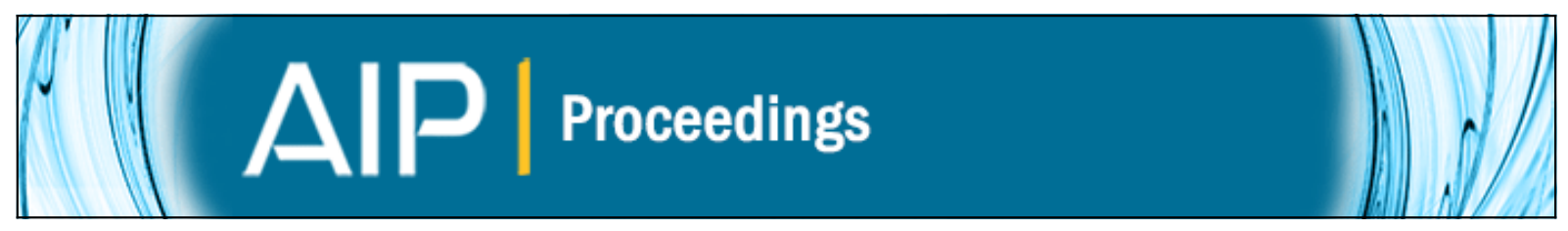

The development of a position-sensitive CZT detector with orthogonal co-planar anode strips

K. A. Larson, L. Hamel, V. Jordanov, J. R. Macri, M. L. McConnell, J. M. Ryan, O. Tousignant, and A. Vincent

Citation: AIP Conference Proceedings 510, 774 (2000); doi: 10.1063/1.1307035

View online: http://dx.doi.org/10.1063/1.1307035

View Table of Contents: http://scitation.aip.org/content/aip/proceeding/aipcp/510?ver=pdfcov

Published by the AIP Publishing

\section{Articles you may be interested in}

Gamma ray astronomy

AIP Conf. Proc. 516, 195 (2000); 10.1063/1.1291477

Position sensitive germanium detectors for the Advanced Compton Telescope

AIP Conf. Proc. 510, 794 (2000); 10.1063/1.1303307

Modelling of CZT strip detectors

AIP Conf. Proc. 510, 769 (2000); 10.1063/1.1303303

High altitude balloon flights of position sensitive CdZnTe detectors for high energy X-ray astronomy

AIP Conf. Proc. 510, 764 (2000); 10.1063/1.1303302

Extra-Solar astrophysics with the High Energy Solar Spectroscopic Imager (HESSI)

AIP Conf. Proc. 510, 671 (2000); 10.1063/1.1303285 


\title{
The Development of a Position-Sensitive CZT Detector with Orthogonal Co-Planar Anode Strips
}

\author{
K.A.Larson ${ }^{\mathrm{a}}$, L.Hamel ${ }^{\mathrm{b}}$, V.Jordanov ${ }^{\mathrm{c}}$, J.R.Macri ${ }^{\mathrm{a}}$, M.L.McConnell ${ }^{\mathrm{a}}$, \\ J.M.Ryan ${ }^{\mathrm{a}}$, O.Tousignant ${ }^{\mathrm{b}}$, A.Vincent ${ }^{\mathrm{b}}$ \\ ${ }^{\text {a }}$ Space Science Center, University of New Hampshire, Durham, NH, USA \\ ${ }^{\mathrm{b}} \mathrm{GCM}$, Physics Department, University of Montreal, Montreal, CA \\ ${ }^{\circ}$ Yantra, 12 Cutts Rd., Durham, NH, USA
}

\begin{abstract}
We report on the simulation, construction, and performance of prototype $\mathrm{CdZnTe}$ imaging detectors with orthogonal coplanar anode strips. These detectors employ a novel electrode geometry with non-collecting anode strips in one dimension and collecting anode pixels, interconnected in rows, in the orthogonal direction. These detectors retain the spectroscopic and detection efficiency advantages of single carrier (electron) sensing devices as well as the principal advantage of conventional strip detectors with orthogonal anode and cathode strips, i.e. an $\mathrm{Nx} \mathrm{N}$ array of imaging pixels are with only $2 \mathrm{~N}$ electronic channels. Charge signals induced on the various electrodes of a prototype detector with $8 \times 8$ unit cells $(1 \times 1 \times 5$ $\mathrm{mm}^{3}$ ) are compared to the simulations. Results of position and energy resolution measurements are presented and discussed.
\end{abstract}

\section{INTRODUCTION}

One of the limiting technologies of hard X-ray and gamma-ray imaging is that of simultaneous spectroscopy and imaging. The requirements for high resolution images with good spectral resolution in an efficient and compact design drives research in monolithic or arrays of position-sensitive, spectroscopic detectors. A leading candidate is CsZnTe strip detectors. They offer high atomic number and density stopping power, good spectral resolution and good spatial resolution in a strip detector format without the need for cryogenic cooling. We describe here the advances made with a new design that satisfies many of these criteria.

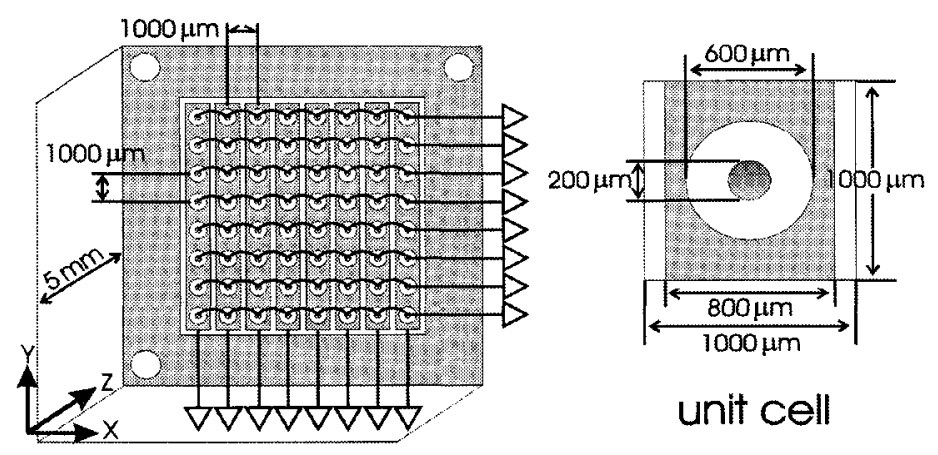

FIGURE 1. Schematic layout showing the prototype coplanar anode detector design and a close up of a unit cell.

CP510, The Fifth Compton Symposium, edited by M. L. McConnell and J. M. Ryan (1) 2000 American Institute of Physics 1-56396-932-7/00/\$17.00 


\section{DETECTOR DESIGN}

CdZnTe (CZT) detectors have faced two major limitations. Pixellated CZT detectors have demonstrated good energy and spatial resolution, but require $\mathrm{n}^{2}$ electronic channels, where $\mathrm{n}$ is the number of pixels in one dimension. Traditional strip detectors reduce the number of electronic channels to $2 \mathrm{n}$, but the poor hole transport properties limit the effective energy range of the device. The detector presented here (figure 1) overcomes both problems by employing a new coplanar anode geometry. An array of anodes (pixels) collects the electron current in this design. Rows of pixels parallel to the $\mathrm{x}$ axis are interconnected providing the $\mathrm{y}$ coordinate of a $\gamma$-ray interaction. Strips parallel to the $y$-axis circumscribe the pixels as shown, but collect no real charge. They are biased at a level between that of the cathode and the pixels, and see only the induced signal generated by electrons drifting towards the collecting pixels. The pixels therefore provide the energy and one coordinate of the position, while the strips provide the other coordinate. This eliminates any dependence on the signal generated by the holes, and allows all electrical connections to be made on one side of the detector.

A low temperature polymer flip chip bonding process was used for the first time to form the electrical and mechanical connection for the patterned CZT and ceramic substrates of our prototype (1). The result is a rugged assembly with no wire bonds to the CZT anode surface. We demonstrated the applicability of our prototype for balloon or space flight in thermal cycle and vibration tests. The results presented were obtained using inexpensive, multi-crystal, counter grade CZT material from eV Products.

\section{LABORATORY MEASUREMENTS}

For these measurements, the pixels were set at zero potential, while the cathode, strips, and guard ring all have adjustable potentials. All output signals are AC-coupled to Ev-5093 charge sensitive preamplifiers with a sensitivity of $3.6 \mathrm{mV} / \mathrm{fC}$. For pulseheight measurements, the signals are processed with standard CAMAC lab equipment. For signal shape analysis, the signals were sampled with digital oscilloscopes (Tektronix TDS360) and then stored for later analysis.

Measurements with ${ }^{57} \mathrm{Co}$ and ${ }^{137} \mathrm{Cs}$ showed a non-uniform response across all pixels. We down-selected a $3 \times 3$ region of cells that produced good and uniform signals. We used the cell in the middle of this region for all measurements. With this cell, the bias levels were varied for the best energy resolution. This meant having full collection on the pixels without significant pixel to strip leakage currents. A cathode bias of -800 Volts with a strip and guard ring bias of -30 to -70 Volts produced the best results.

Energy resolution measurements using a $0.5 \mu$ s gaussian shaping time were made at room temperature with events triggering only from the cell under test. Figure 2 shows the spectra from two $\gamma$-ray sources, along with the pulser for a noise estimate. 
For ${ }^{137} \mathrm{Cs}$, the FWHM is $5.9 \mathrm{keV}$, or $0.9 \%$, while the pulser FWHM is $3.3 \mathrm{keV}$. For ${ }^{241} \mathrm{Am}$, the resolution is $3.4 \mathrm{keV} \mathrm{FWHM}$, or $5.7 \%$. If the electronic noise from the pulser is quadratically subtracted from the resolution, an intrinsic resolution of $0.7 \%$ has been achieved at $662 \mathrm{keV}$.
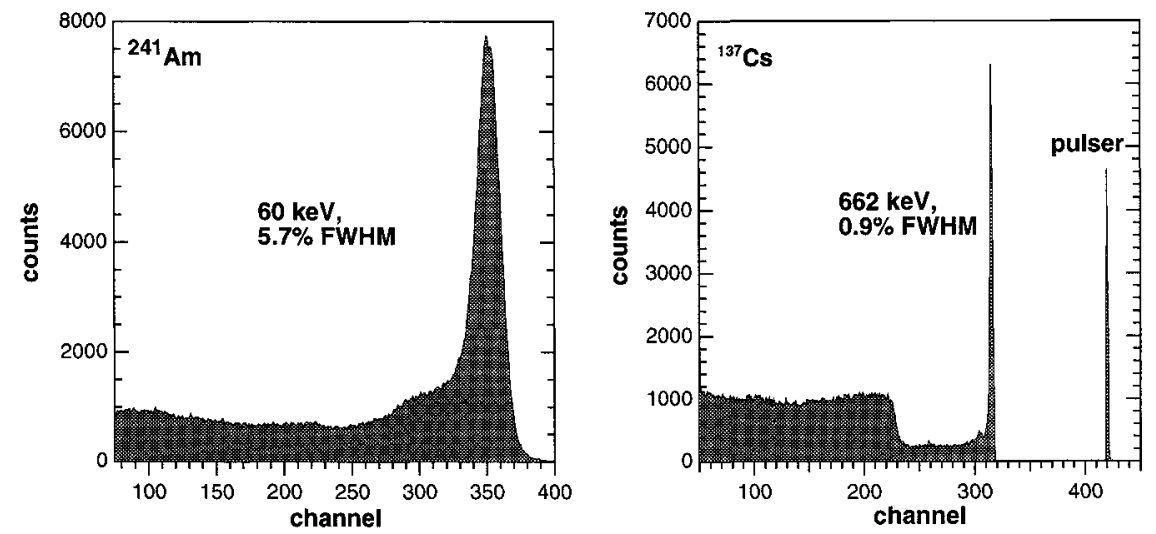

FIGURE 2. Energy spectra for ${ }^{241} \mathrm{Am}$ and ${ }^{137} \mathrm{Cs} \gamma$-ray sources.
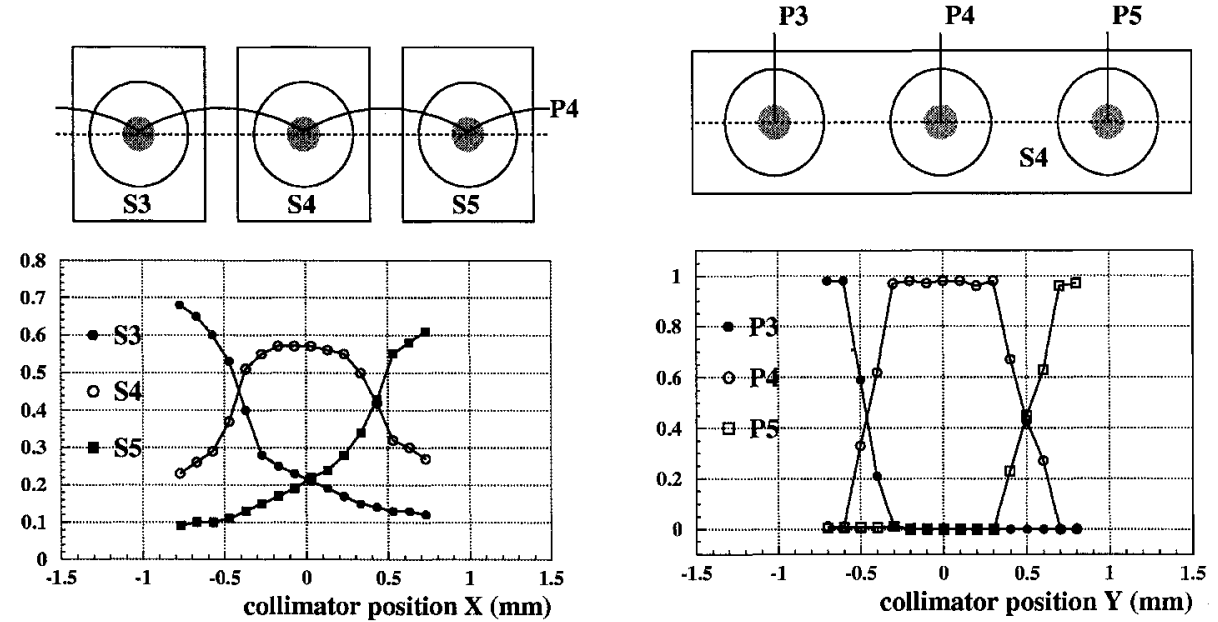

FIGURE 3. Collimated $\alpha$-ray scans. For both plots, the relative amplitudes on three adjacent channels (normalized to their sum) are plotted as a function of source position. The X-scan plot (left) shows very little charge sharing between pixels, while the Y-scan plot (right) shows greater sharing.

For position resolution measurements, a collimated ${ }^{244} \mathrm{Cm} \alpha$ source on a 3-axis micro-manipulator illuminated a spot approximately $200 \mu \mathrm{m}$ in diameter. The source was scanned across the $3 \times 3$ cell area and events for each pixel and strip were recorded on the digital oscilloscopes. The center cell was scanned to determine the location of the maximum signal with the signal of its neighboring cells being roughly equal. Since 
we could not see the location of the spot illuminated with $\alpha$ 's, we took this location to be the center of the cell. The $3 \times 3$ region was then scanned in $x$ and $y$ in $100 \mu \mathrm{m}$ steps, and the pulse heights were recorded. The results of the scan, using mean amplitudes normalized for each event, are shown in figure 3 .

While significant charge sharing occurs between strips, little charge sharing occurs between pixels. What there is is limited to a small region directly between the pixels (figure 3). As a result, the analysis of relative pixel pulse heights yields little information. Consequently, the resolution in y is on the order of $1 \mathrm{~mm}$. For strips, however, position information is shared among as many as three strips. The analysis of the relative pulse heights of those strips yields a resolution on the order of $300 \mu \mathrm{m}$.

The measured signals have been compared to simulations (2). Figure 4 shows simulation results compared to scope traces for ${ }^{137} \mathrm{Cs} \gamma$-rays interacting at three different depths. Three events were selected for which signals on neighboring strips were equal, ensuring that all three occurred at the center of the cell. Measured signals were matched with simulation signals to determine the approximate depth of interaction.

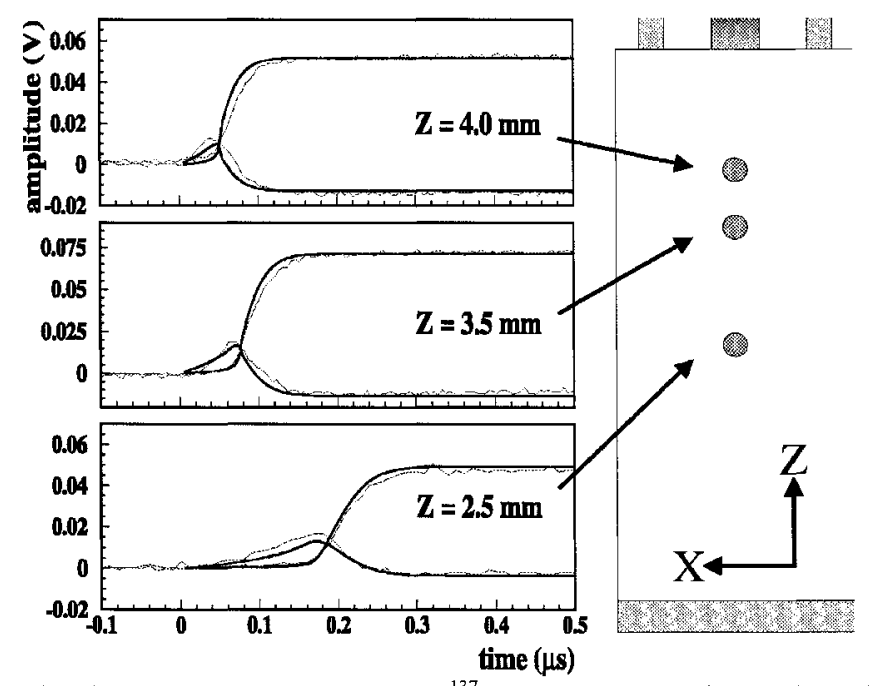

FIGURE 4. Simulated and measured signals from ${ }^{137} \mathrm{Cs} \quad \gamma$-rays interacting at three different depths. The depth position is inferred from the simulation.

It is clear from the simulation results that the strip signal shape carries information about the depth of interaction. Both the rise time and residual level vary with depth. Figure 5 shows these parameters plotted against one another for both simulated and measured events. The scatter in the points indicates a $z$ position resolution of approximately $1 \mathrm{~mm}$. Figure 5 also shows a plot of the strip residual versus depth for ${ }^{137} \mathrm{Cs} \gamma$-rays for both simulated and measured events using the normalized large amplitude cathode signal as a measure of depth. Depth information can also be gained strictly from examination of the cathode signal, but due to poor hole mobility the main 
signal component is induced charge from the movement of electrons. Small events far away from the cathode may therefore not produce signals above the noise.
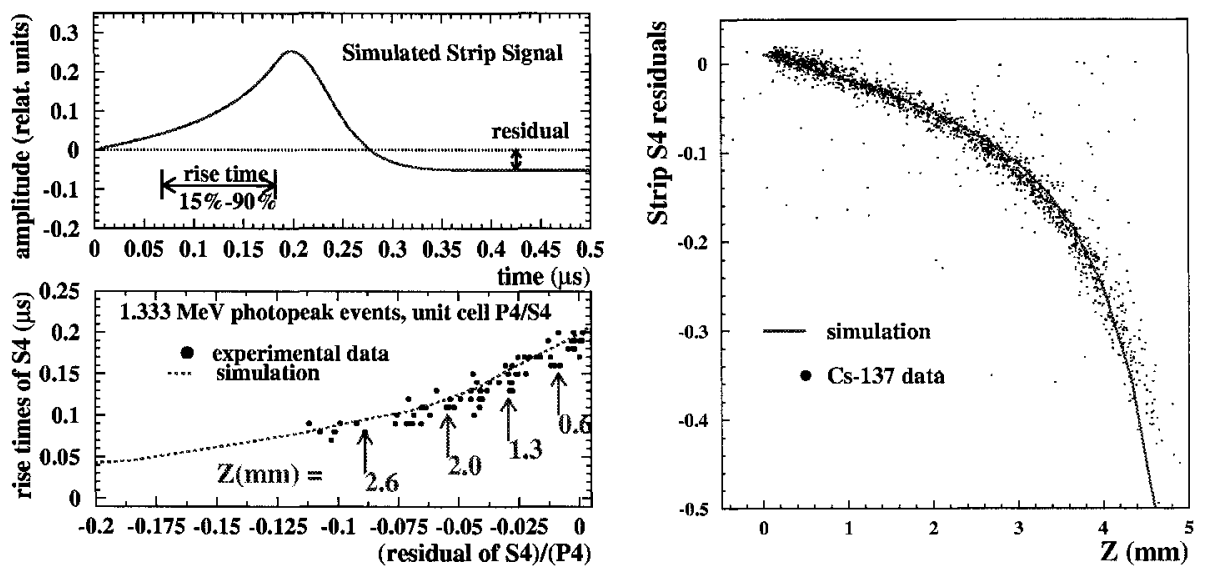

FIGURE 5. Depth of interaction derived from measurement of strip signal risetime and residual, both measured and simulated, for $1.333 \mathrm{MeV}$ (left). Depth of interaction derived solely from strip signal residual for ${ }^{137} \mathrm{Cs} \gamma$-rays, both measured and simulated (right).

\section{CONCLUSIONS}

A prototype of a CZT imaging detector with orthogonal coplanar anodes has been fabricated. Tests have confirmed that this detector could provide excellent energy resolution and imaging performanc. Signals observed on both pixels and strips are in good agreement with the simulations. Future work will include complete characterization of the detector response, as well as imaging with a 16 channel digital acquisition system currently in developement.

\section{ACKNOWLEDGEMENTS}

This work is supported by NASA's High Energy Astrophysics SR\&T program and by the Natural Sciences and Engineering Research Council (NSERC) of Canada.

\section{REFERENCES}

1. Jordanov, V.T., et al., "Multi-Electrode CZT Detector Packaging Using Polymer Flip Chip Bonding," presented at $11^{\text {th }}$ International Workshop on Room Temperature Semiconductor Xand Gamma-Ray Detectors and Associated Electronics, Vienna, Austria, October 1999.

2. Hamel, L.A., et al., "An Imaging CdZnTe detector with coplanar orthogonal strips", Proceedings of the 1997 Fall Meeting of the Materials Research Society, Boston, 1-5 December, 1997. 